\title{
U.S. Landsat Analysis Ready Data
}

U.S. Landsat Analysis Ready Data (ARD) has revolutionized how Landsat data are processed. U.S. Landsat ARD significantly reduces the burden of preprocessing of Landsat data, allowing users to easily produce Landsatbased maps of land cover and land-cover change and other derived geophysical and biophysical products.

U.S. Landsat ARD contains Level-2 products derived from Landsat Collections Level-1 scenes. ARD is provided as tiled, georegistered, top of atmosphere, and atmospherically corrected products defined in a common equal area projection for immediate use in monitoring and assessing landscape change.

U.S. Landsat ARD is available for the conterminous United States, Alaska, and Hawaii, using the following Landsat Collections Level-1 products:

- Landsat 8 Operational Land Imager (OLI)/Thermal Infrared Sensor (TIRS) Tier 1, Tier 2

- Landsat 7 Enhanced Thematic Mapper Plus (ETM+) Tier 1

- Landsat 4-5 Thematic Mapper (TM) Tier 1

\section{Products}

U.S. Landsat ARD consists of the following products and spatially explicit quality assessment information to document the presence of sensor, solar, atmospheric and topographic conditions and traceability to the Landsat Level-1 input source scenes used in ARD:

- Top of Atmosphere (TA) reflectance (reflectance calculated "at sensor," not atmospherically corrected)

- Top of Atmosphere Brightness Temperature (BT) (radiance calculated "at sensor," not atmospherically corrected, expressed in Kelvin)

- Surface Reflectance (SR) (atmospherically corrected)

- Surface Temperature (ST) (temperature of the Earth's surface, expressed in Kelvin)

- Pixel Quality Assessment (QA) (common and sensorspecific quality bands included)

U.S. Landsat ARD products are generated in the Albers Equal-Area (AEA) Conic map projection, processed directly from Landsat Level-1 AEA scenes through the Landsat Level-2 product generation system using the World Geodetic System 1984 (WGS 84) datum.

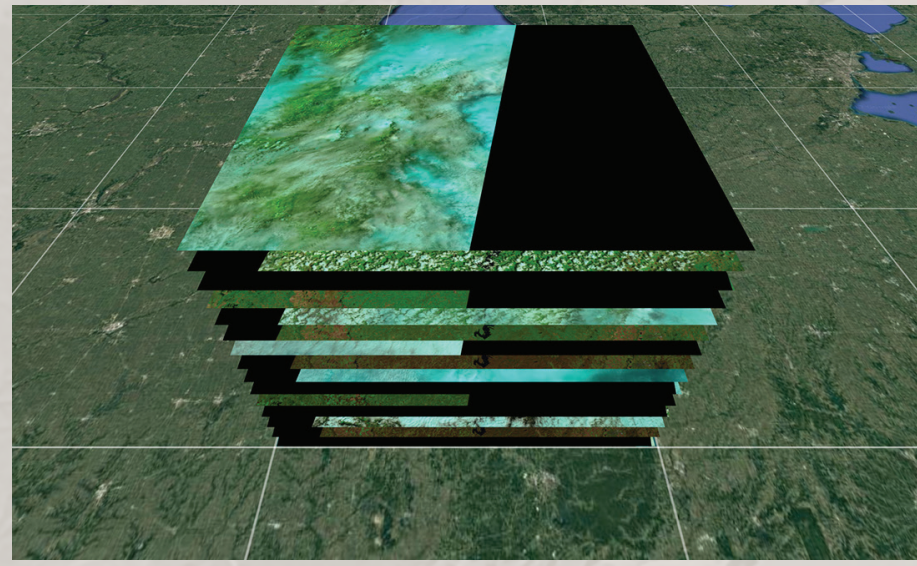

Figure 1. U.S. Landsat Analysis Ready Data sample stack of one tile. Background imagery courtesy of GoogleEarth.

\section{Tile Grid System}

U.S. Landsat ARD products are processed into spatially consistent tiles of data, modified from the Web Enabled Landsat Data (WELD) grid system. The WELDdefined grid is similar to the National Land Cover Database (NLCD), except that WELD is based on the WGS 84 datum and NLCD uses the North American Datum of 1983 (NAD 83). The difference in datum causes an approximate 0.5 -pixel offset in the $\mathrm{x}$ and $\mathrm{y}$ directions between the two grids. The U.S. Landsat ARD projection parameters are listed in table 1.

Projection: Albers Equal-Area Conic

Datum: World Geodetic System 1984 (WGS 84)

Output format: Georeferenced Tagged Image File Format (GeoTIFF)

Table 1. U.S. Landsat Analysis Ready Data projection parameters.

$\left[{ }^{\circ}\right.$, degree $]$

\begin{tabular}{lcrr}
\hline \multicolumn{1}{c}{ Projection parameter } & $\begin{array}{r}\text { Conterminous } \\
\text { United States }\end{array}$ & Alaska & Hawaii \\
\hline First standard parallel & $29.5^{\circ}$ & $55.0^{\circ}$ & $8.0^{\circ}$ \\
\hline Second standard parallel & $45.5^{\circ}$ & $65.0^{\circ}$ & $18.0^{\circ}$ \\
\hline Longitude of central meridian & $-96.0^{\circ}$ & $-154.0^{\circ}$ & $-157.0^{\circ}$ \\
\hline Latitude of projection origin & $23.0^{\circ}$ & $50.0^{\circ}$ & $3.0^{\circ}$ \\
False easting (meters) & 0.0 & 0.0 & 0.0 \\
\hline False northing (meters) & 0.0 & 0.0 & 0.0 \\
\hline
\end{tabular}


Each tile contains $5,000 \times 5,00030$-meter Landsat pixels that are acquired on a given day. The U.S. Landsat ARD tile pixel size is smaller than a traditional Landsat scene (Landsat 8 is about $6,300 \times 6,000$ pixels; Landsat 7 is about $6,166 \times 5,666$ pixels; fig. 2 ). This tiling scheme ensures that each pixel in a U.S. Landsat ARD tile represents the same location on the Earth's surface through the entire U.S. Landsat ARD time series record from 1982 to the present.

- 422 tiles cover the land mass extent of the conterminous United States,

- 110 tiles cover the land mass extent of Alaska, and

- 8 tiles cover the islands of Hawaii.

Figure 2. U.S. Landsat Analysis Ready Data tile h05v02 overlaid on Landsat 8 Worldwide Reference System 2 (WRS-2) path 44 row 27 for illustrative purposes. (Landsat ARD product identifier: LC08_CU_005002_20130603_20170713_C01_V01, Landsat product identifier: LC08_L1TP_044027_20130603_20170310_01_T1).

\section{Product Packaging, Filenames, and Accessibility}

U.S. Landsat ARD provides users with notable flexibility in how they access customized Landsat data. For example, users have the ability to tailor data queries according to application needs in terms of geospatial regions, spectral bands and collection dates.

U.S. Landsat ARD products are packaged into bundles and delivered as .tar files. The sizes of the U.S. Landsat ARD bundles vary greatly and have the potential of being substantially larger than the traditional Landsat Level-1 scene-based products.

U.S. Landsat ARD products are available for download at no charge from EarthExplorer at https://earthexplorer.usgs.gov/.

\section{For More Information}

For additional information or to learn more about U.S. Landsat ARD, please visit the U.S. Landsat ARD web page at https://www.usgs.gov/land-resources/nli/landsat/ us-landsat-analysis-ready-data.

Questions about Landsat operations, data products, and data access can be directed to

Landsat User Services

U.S. Geological Survey

Earth Resources Observation and Science (EROS) Center 47914 252d Street

Sioux Falls, SD 57198

Phone: 605-594-6151 or 1-800-252-4547

Email: custserv@usgs.gov

Twitter:@USGSLandsat

Facebook: https://www.facebook.com/NASA.Landsat

Visit https://www.usgs.gov for more information about the USGS and https://www.usgs.gov/land-resources/nationalland-imaging-program for specifics about the National Land Imaging Program. 